\title{
Demonstration of Three Dimensional Imaging of Blood Vessel using a No-Moving Parts Electronic Lens-based Optical Confocal Microscope
}

\author{
Nabeel A. Riza ${ }^{* a}$, Mumtaz Sheikh ${ }^{\mathrm{a}}$, Grady Webb-Wood ${ }^{\mathrm{b}}$, and Pieter Kik ${ }^{\mathrm{b}}$ \\ ${ }^{\mathrm{a}}$ Photonic Information Processing Systems (PIPS) Laboratory \\ ${ }^{\mathrm{b}}$ Nano-Photonics and Near Field Optics Laboratory \\ The College of Optics \& Photonics/CREOL \\ University of Central Florida \\ 4000 Central Florida Blvd., Orlando, FL 32816-2700 \\ *email: riza@creol.ucf.edu Tel: 407-823-6829 Fax: 407-823-6880
}

\begin{abstract}
To the best of our knowledge, for the first time, biological Three Dimensional (3-D) imaging has been achieved using an electronically controlled optical lens to accomplish no-moving parts depth section scanning in a modified commercial 3D confocal microscope. Specifically, full 3-D views of a standard CDC blood vessel (enclosed in a glass slide) have been obtained using the modified confocal microscope operating at the red $633 \mathrm{~nm}$ laser wavelength.
\end{abstract}

Keywords: Optical imaging, microscopic imaging, confocal microscopy, no-moving parts.

\section{INTRODUCTION}

Confocal microscopes are widely used for 3-D imaging of microscopic objects. In the basic confocal microscope, a point source is focused onto the sample using an objective lens. The reflected or transmitted light, depending on which configuration is used, is collected by the same or a different objective lens and focused on a tiny pinhole in front of the detector using a spherical lens. The size of the pinhole is chosen to let only the central part of the Airy pattern to hit the detector. This ensures that only the rays coming from the image focal plane can reach the detector while all the out-offocus light is rejected. This confocal process strongly increases image contrast as well as slightly improves axial resolution as compared to conventional optical microscopes [1-4].

Present-day confocal microscopes use piezoelectric mechanical motion stages for 3-D scanning of test samples to realize full 3-D views. As piezoelectric stages have inherent hysteresis issues, the system uses complex feedback controls to maintain scan motion operational accuracy. In effect, these all-mechanical scan confocal microscopes become complex, power hungry, bulky, and expensive systems. Recently, we proposed the use of electronic optical scanning technology to alleviate this moving parts scanning burden for commercial confocal microscopes [5]. To our knowledge, this is the first time that Electronic (E) lens technology using liquid crystals has been applied to full 3-D view confocal microscopy and produced 3-D images of blood vessel biological samples.

\section{PROPOSED E-LENS BASED AXIAL SCANNING CONFOCAL MICROSCOPE}

We have recently shown how liquid crystal lens technology can be used in a basic confocal microscopy set-up to enable no-moving parts axial scanning to realize depth direction scans of electronic and optical materials to produce material one dimensional profile sections [6]. The focus of the present paper is to show how our earlier developed electronic lensbased confocal design can be applied to a full 3-D scan commercial confocal microscopy system, a WITec AlphaSNOM, in order to realize a next generation unit where depth scanning is achieved via a no-moving parts device (see Fig.1). More specifically, we apply the technique to biological sample imaging to benefit medical arena users. The proposed approach uses the concept of programmable weak thin lens cascading with a strong fixed focal length microscope objective lens to form a variable focal length objective.

Medical Imaging 2007: Physics of Medical Imaging, edited by Jiang Hsieh, Michael J. Flynn, Proc. of SPIE Vol. 6510, 65100J, (2007) · 1605-7422/07/\$18 · doi: 10.1117/12.708836 


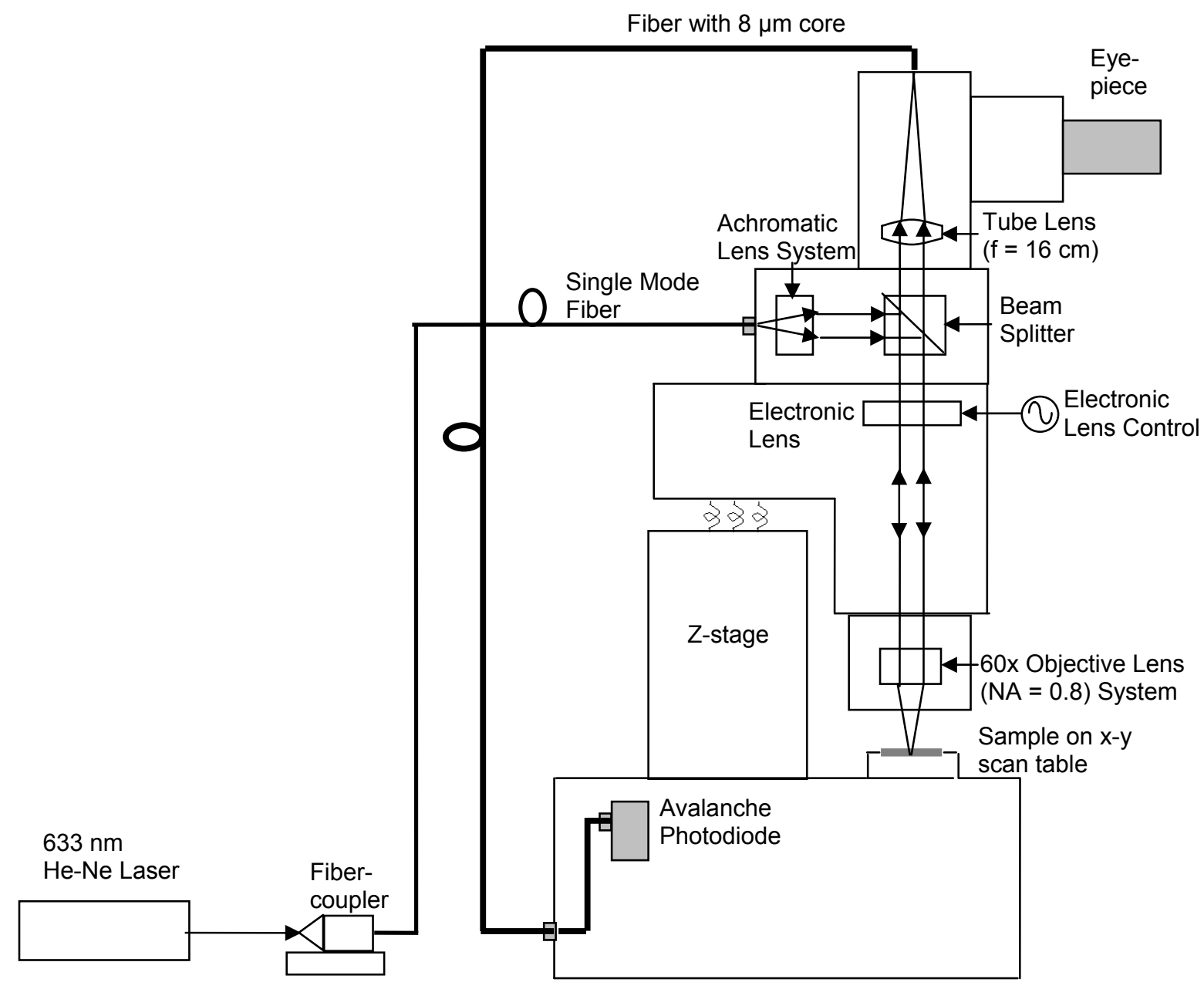

Fig.1. Proposed electronic lens-based axial scanning full 3-D imaging confocal microscope systems realized by modifying a WITec AlphaSNOM system.

\section{EXPERIMENTAL DEMONSTRATION}

Fig. 2 shows a cross-sectional view and dimensions of an approximately two months old pig artery in a Center for Disease Control (CDC) slide No.IHC04-115, Hemediagnostic S-100D. 3-D images of the pig artery have been realized using both the conventional all-mechanical scanning confocal microscope and the modified electronic lens-based confocal microscope. Fig.3 shows transverse (or cross-sectional) views of the Fig.2 marked square targeted area. The scans in Fig. 3 are 500 pixel x 500 pixel with motion step of 0.1 microns enabling a 50 micron x 50 micron imaged zone. The electronic lens and mechanical motion axial scans are implemented over 20 microns that fully covers the artery sample axial size. Fig.3(a) data is obtained by using the mechanical z-stage motion to position the confocal depth at a chosen position within the artery. Next, with the mechanical z-stage at the same location, the electronic lens is turned on for a 5 microns focal shift and a transverse scan is taken (see Fig 3(b)). The change in focus caused by the electronic lens is clearly visible. With the electronic lens still on, Fig.3(c) data is obtained by moving the mechanical z-stage by a distance of 5 microns to recover the desired known axial location and then implement a mechanical cross-sectional scan. Fig.3(a) and Fig.3(c) views are similar indicating that the electronic lens-based 3-D confocal microscope is an effective tool for imaging. 


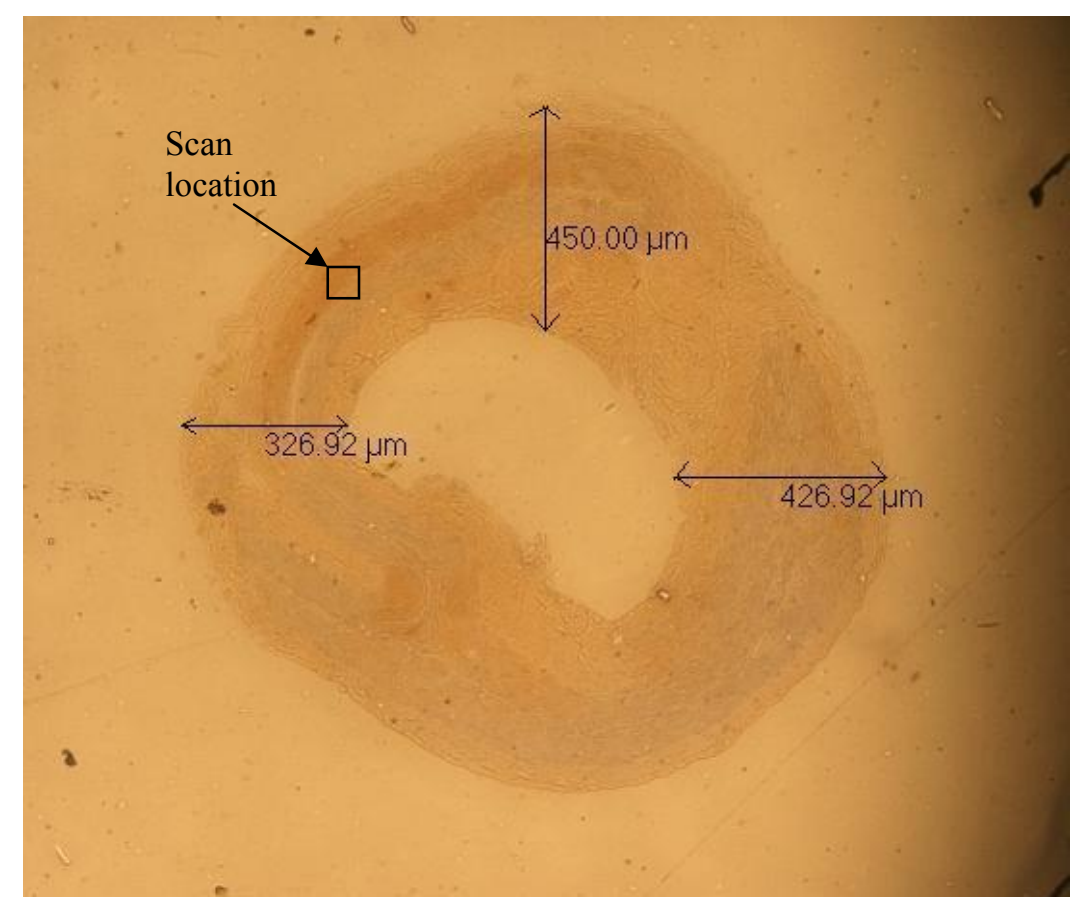

Fig.2. Cross-Section of Pig Artery from CDC Slide produced using standard optical microscope.

The electronic lens is based on nematic liquid crystal technology [7] and enables microscope objective focal length changes via adjustment of the electronic lens drive frequency. For comparison, full 3-D views of the blood vessel were generated using the mechanical depth scanning stages built into the commercial microscope. Our study shows that the electronic lens-based 3-D views match the standard mechanical operation results. 50 micron x 50 micron size 500 pixel $\mathrm{x}$ 500 pixel image slices were obtained with pixel or physical motion step of 0.1 micron. Electronic and mechanical depth or axial direction scanning was achieved over a 20 micron zone more than adequate to scan the pig artery. Because traditional piezo-electric mechanical motion stages have hysteresis issues and require complex feedback systems, leading to a complex, bulky, and costly system, the proposed electronic lens-based microscope can alleviate some of the burden on the microscope control electronics, in addition to improving long-term reliability via the no-moving parts design. Also, imaging of biological samples can be faster using this electronic lens based acquisition process versus the inertialimited translation stage, enabling capture of fast temporal effects. 


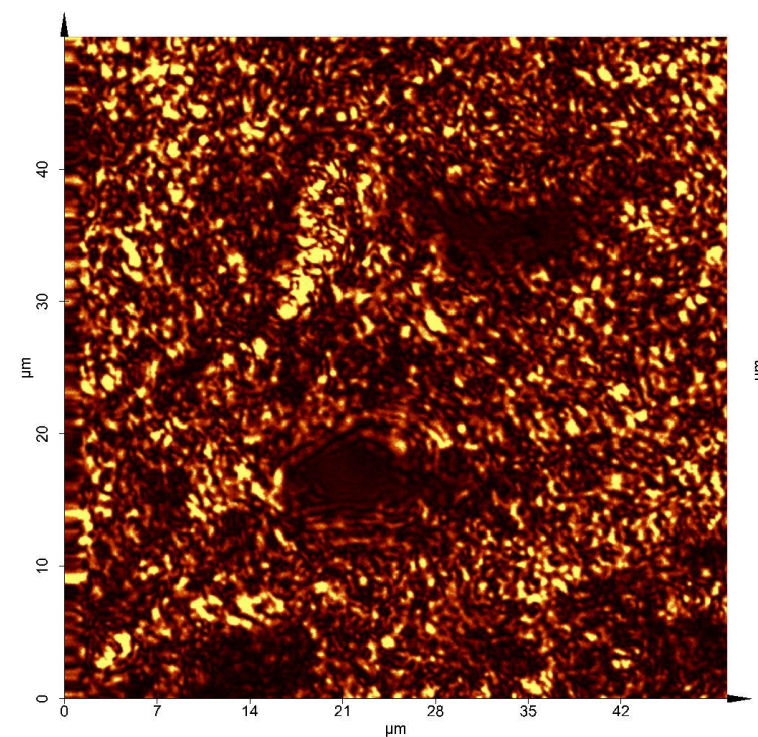

(a)

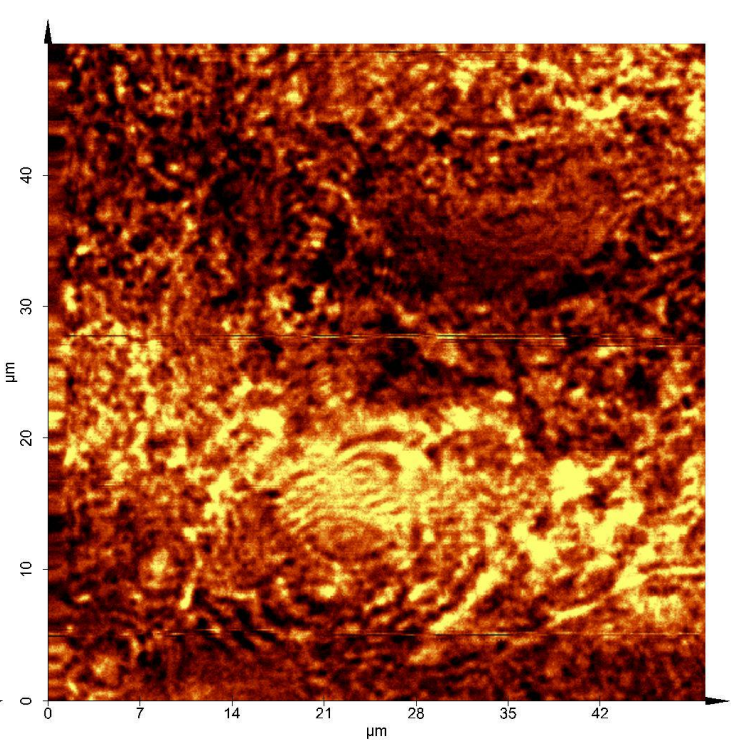

(b)

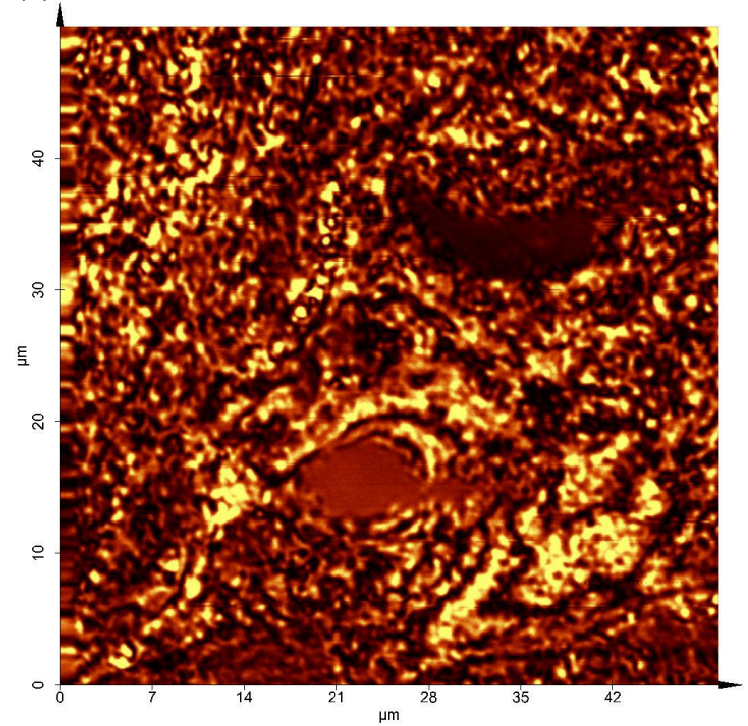

(c)

Fig.3. Artery sample $50 \mu \mathrm{m}$ x $50 \mu \mathrm{m}$ cross-sectional or transverse (x-y) scan of 500 pixels x 500 pixels using (a) Conventional All-Mechanical Scan Confocal Microscope, (b) Modified Confocal Microscope using Electronic lens set for a 5 micron focal length change, and (c) Modified Confocal Microscope using 5 micron focus change on-state Electronic Lens and a counter-direction 5 micron axial/depth scan position offset via mechanical motion to recover Fig 3(a) image.

\section{CONCLUSION}

We have successfully shown that electronic lens technology can be applied to conventional confocal microscopy systems to realize a no-moving parts axial scanning microscope with reasonably equivalent 3-D imaging results when applied to biological samples. Improved optical designs can reduce optical aberrations in the proposed E-lens based confocal microscope. Just like the standard confocal microscope, this technique would also work equally well in a fluorescence microscope arrangement [8]. In the future, one can envision a confocal microscope with complete 3-D scanning accomplished using a no-moving parts design, allowing power and space efficient fast 3-D imaging of real-time biological effects. 


\section{ACKNOWLEDGEMENTS}

We thank Dr. Sajjad Khan for initial experimental support. In addition, we thank Prof. C. Yang of Caltech for providing the CDC blood vessel slide.

\section{REFERENCES}

1. M. Minsky, “Microscopy Apparatus,” US Patent 3013467, 1961.

2. T. Wilson, Confocal Microscopy, Academic Press, San Diego, 1990.

3. T. Corle and G. Kino, "Confocal Scanning Optical Microscopy and Related Imaging Systems," Academic Press, San Diego, 1996.

4. M. Rajadhyaksha, R. Anderson, and R. Webb, "Video-Rate Confocal Scanning Laser Microscope for Imaging Human Tissues In Vivo,” Applied Optics, Vol. 38, Issue 10, Pages 2105-2115, April 1999.

5. N. A. Riza and A. Bokhari, "Agile Optical Confocal Microscopy Instrument Architectures For High Flexibility Imaging," in Three Dimensional Confocal Microscopies, BIOS 2004 Biomedical Optics, Photonics West, Proc. SPIE Vol. 5324, Paper No. 14, Pages 77-88, January 2004, San Jose, California, USA.

6. S. A. Khan and N. A. Riza, "Demonstration of a No-Moving-Parts Axial Scanning Confocal Microscope Using Liquid Crystal Optics," Optics Communications, Vol. 265, Issue 2, Pages 461-467, September 2006.

7. G. Vdovin, A. Naumov, M. Loktev, V. Belopukhov, F. Vladimirov, and G. Love, " Wave front control systems based on modal liquid crystal lenses,” Rev. Sci. Instrum., Vol. 71, Issue 9, Pages 3290-3297, 2000.

8. F. Rost and R. Oldfield, Fluorescence microscopy, Photography with a Microscope, Cambridge University Press, Cambridge, United Kingdom, 2000. 